\title{
Telemedicine Expansion During the COVID- 19 Pandemic and the Potential for Technology-Driven Disparities
}

J Gen Intern Med 36(1):256-8

DOI: $10.1007 / \mathrm{s} 11606-020-06322-\mathrm{y}$

(c) Society of General Internal Medicine 2020

\section{BACKGROUND}

Telemedicine use has rapidly increased across the US health system during the COVID-19 pandemic. ${ }^{1}$ Although telemedicine has been heralded as a way to reduce disparities in healthcare, ${ }^{2}$ concerns remain that lack of access to technology or digital health literacy can exacerbate technology-driven disparities as telemedicine use expands. ${ }^{2-4}$ As recent regulatory and policy changes allowed reimbursement for telephonic telemedicine visits in addition to visits facilitated by audiovideo technology, ${ }^{1}$ there is a unique opportunity to examine technology-driven disparities as manifested through how telemedicine services are accessed differently by different patient populations.

\section{OBJECTIVE}

We sought to assess disparities in whether patients received audio-video telemedicine visits or telephonic ones, using data from a telemedicine expansion initiative at a major academic medical center.

\section{METHODS AND FINDINGS}

Beginning on March 2, 2020, the Columbia University Irving Medical Center undertook rapid expansion of telemedicine services across all outpatient clinical services through centralized training and support and increased patient outreach and education. All departments were strongly recommended to conduct telemedicine visits through Epic EHR (Epic; Verona, WI) integrated audio-video technology (Vidyo; Hackensack, NJ), but could also use telephone visits if necessary (e.g., if patients lacked smartphone or internet access).

We queried Epic EHR for all scheduled outpatient telemedicine visits completed over a 13-week period from February 1, 2020, to May 1, 2020, using visit types and scheduling comments to define audio-video versus telephone visits. We

Received June 24, 2020

Accepted October 15, 2020

Published online October 26, 2020 collected patient demographic information (age, sex, race, and ethnicity) and visit information (specialty, clinic site, and primary insurance). We used descriptive statistics to summarize the number of telemedicine visits over time. A multi-level logistic regression model was used to estimate the odds of having a telemedicine visit through audio-video technology versus telephone, accounting for specialties as fixed effects and clinic sites as random effects. We applied inverse weighting to account for multiple patient visits at practice level.

From February 1 to May 1, 2020, 50,101 unique patients (Table 1) received a total of 80,163 telemedicine visits, including $60,712(76 \%)$ visits conducted through audio-video and 19,411 (24\%) conducted via telephone. The weekly number of telemedicine visits increased steadily, from 56 during week 1 to 13,985 during week 13 (Fig. 1).

In the fully adjusted model, after accounting for specialty area and clinic sites, older age, Black race, Hispanic ethnicity or primary language Spanish, and primary insurance being Medicaid or Medicare were all significantly associated with lower odds of audio-video telemedicine visits (Table 1).

\section{DISCUSSION}

Our successful telemedicine expansion in response to COVID-19 is consistent with similar reports from across the USA. ${ }^{5}$ However, despite increased coverage for telemedicine services and additional infrastructure and personnel support, we found that older patients, minorities, and patients with public insurance are less likely to receive telemedicine services through audio-video technology. As previous research suggests that telephone visits are less effective for patient communication and comprehension, these disparities may further negatively impact patient care. ${ }^{6}$

Our findings are consistent with prior studies showing age and race disparities in usage of health information technology such as patient portals. ${ }^{4}$ As telemedicine will remain an important aspect of the US healthcare delivery for the foreseeable future, our findings also have immediate policy implications for telemedicine services. To support telemedicine expansion, CMS has granted flexibility for the use of non-EHR-based audio-video platforms (such as FaceTime and Skype) and has increased reimbursement for telephone visits. ${ }^{1}$ Our study suggests that vulnerable patient populations have difficulty 
Table 1 Patient Characteristics and Predictors of Telemedicine Visits Being Conducted Using Audio-Video Technology Versus Telephone Only. Model Adjusted for Specialties as Fixed Effects and Clinic Sites as Random Effects, and Accounted for Multiple Visits by Same Patient at the Practice Level Through Inverse Weighting; Odds Ratios (OR) Lower Than 1 Denotes Lower Odds of Audio-Video Telemedicine Visits

\begin{tabular}{|c|c|c|c|}
\hline Characteristic & Unique patients $(N=50,101)$ & Odds ratio $(95 \% \mathrm{CI})$ & $P$ value \\
\hline \multicolumn{4}{|l|}{ Age range } \\
\hline 0 to 11 & $4382(4.4 \%)$ & $1.20(0.94$ to 1.53$)$ & 0.15 \\
\hline 12 to 17 & $2747(5.5 \%)$ & $0.89(0.66$ to 1.20$)$ & 0.43 \\
\hline 18 to 44 & $17,561(35.1 \%)$ & Ref & Ref \\
\hline 45 to 64 & $12,813(25.6 \%)$ & $0.38(0.32$ to 0.45$)$ & $<0.001$ \\
\hline 65 and above & $12,598(25.2 \%)$ & $0.18(0.14$ to 0.23$)$ & $<0.001$ \\
\hline Female gender & $30,500(60.9 \%)$ & $1.03(0.91$ to 1.16$)$ & 0.65 \\
\hline \multicolumn{4}{|l|}{ Race } \\
\hline White & $21,983(43.9 \%)$ & Ref & Ref \\
\hline American Indian/Alaska Native & $108(0.2 \%)$ & $0.91(0.54$ to 1.52$)$ & 0.72 \\
\hline Asian & $1470(2.9 \%)$ & $1.17(0.91$ to 1.51$)$ & 0.21 \\
\hline Black or African American & $6034(12.0 \%)$ & $0.80(0.72$ to 0.89$)$ & $<0.001$ \\
\hline Native Hawaiian/Pacific Islander & $273(0.5 \%)$ & $0.95(0.65$ to 1.39$)$ & 0.79 \\
\hline Other/Declined to Answer & $20,233(40.4 \%)$ & $0.87(0.80$ to 0.94$)$ & $<0.001$ \\
\hline Hispanic or primary language Spanish & $13,499(26.9 \%)$ & $0.82(0.74$ to 0.91$)$ & $<0.001$ \\
\hline \multicolumn{4}{|l|}{ Primary insurance } \\
\hline Commercial & $26,479(52.9 \%)$ & Ref & Ref \\
\hline Medicaid & $10,990(21.9 \%)$ & $0.57(0.48$ to 0.68$)$ & $<0.001$ \\
\hline Medicare & $12,632(25.2 \%)$ & $0.52(0.44$ to 0.63$)$ & $<0.001$ \\
\hline
\end{tabular}

engaging with audio-video telemedicine visits even in this permissive environment, suggesting that caution is needed when more restrictive policies resume.

Our study has several limitations. We used data from a single, urban academic medical center and did not assess general access to care during the COVID-19 pandemic. Future analyses will also need to address other factors such as provider or scheduler implicit bias and patient comorbidities. There is also potential misclassification of audio-video vs. telephone visits, such as when a visit is scheduled as audiovideo but was completed by telephone. Nonetheless, we are amongst the first to illustrate potential technology-driven disparities resulting from large-scale telemedicine expansion in the USA, highlighting the urgent need to identify policies and interventions to ensure that telemedicine technology can be equitably accessed by patients and does not further exacerbate disparities due to gaps in technology access and digital health literacy.

Siqin Ye, MD, $M S^{1}$

Ian Kronish, $M D, M^{1}$

Elaine Fleck, $M D^{1,2}$

Peter Fleischut, $M D^{2}$

Shunichi Homma, MD, MHCDS ${ }^{1}$

David Masini, BS ${ }^{1}$

Nathalie Moise, $M D, M^{1}$

${ }^{1}$ Columbia University Irving Medical Center, New York, NY, USA

${ }^{2}$ New York Presbyterian Hospital,

New York, NY, USA

Corresponding Author: Siqin Ye, MD, MS; Columbia University Irving Medical Center, New York, NY, USA (e-mail: sy2357@cumc.columbia.edu).

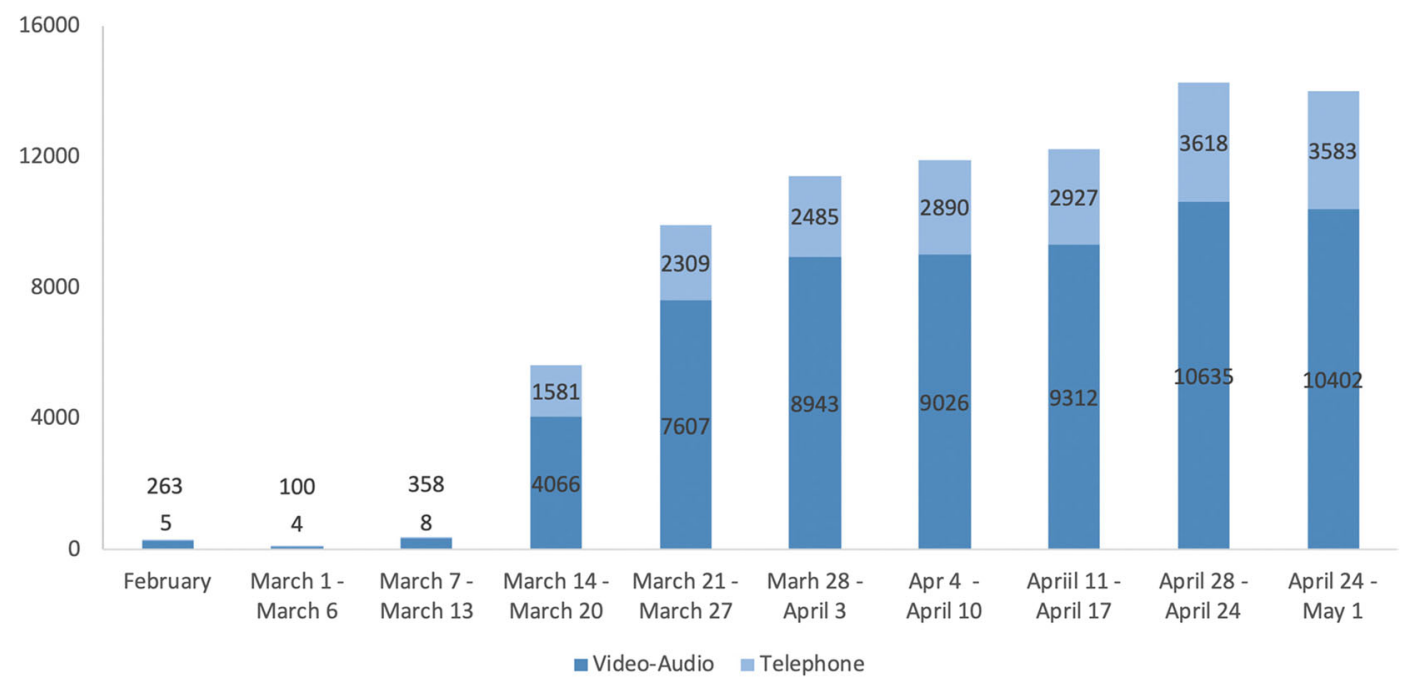

Figure. 1 Number of telemedicine visits from February 1, 2020, to May 1, 2020. 


\section{Compliance with Ethical Standards:}

Conflict of Interest: The authors declare that they do not have a conflict of interest.

\section{REFERENCES}

1. Cutler DM, Nikpay S, Huckman RS. The business of medicine in the era of covid-19. JAMA. 2020;323(20):2003-2004. https://doi.org/10.1001/ jama.2020.7242. Accessed 18 May 2020.

2. Roundtable on the promotion of health equity and the elimination of health disparities; board on population health and public health practice; health and medicine division; national academies of sciences, engineering, and medicine. The promises and perils of digital strategies in achieving health equity: Workshop summary. Washington (dc): National academies press (us); 2016 Jun 22. 2, technology and health disparities. Available from: https://www.Ncbi.Nlm.Nih.Gov/books/nbk373436/. Accessed 15 July 2020.
3. Velasquez D, Mehrotra A. Ensuring the growth of telehealth during covid19 does not exacerbate disparities in care, health affairs blog, May 8, 2020; https://doi.org/10.1377/hblog20200505.591306.

4. Walker DM, Hefner JL, Fareed N, Huerta TR, McAlearney AS. Exploring the digital divide: Age and race disparities in use of an inpatient portal. Telemed J E Health. 2019;26(5):603-613. https://doi.org/10.1089/tmj. 2019.0065. Accessed 15 July 2020.

5. Mann DM, Chen J, Chunara R, Testa PA, Nov O. Covid-19 transforms health care through telemedicine: Evidence from the field. J Am Med Inform Assoc. 2020;27(7):1132-1135. https://doi.org/10.1093/jamia/ ocaa072. Accessed 19 May 2020.

6. Lion KC, Brown JC, Ebel BE, et al. Effect of telephone vs video interpretation on parent comprehension, communication, and utilization in the pediatric emergency department: A randomized clinical trial. JAMA Pediatr. 2015;169(12):1117-1125. https://doi.org/10.1001/ jamapediatrics.2015.2630. Accessed 21 May 2020.

Publisher's Note: Springer Nature remains neutral with regard to jurisdictional claims in published maps and institutional affiliations. 